\title{
Identification of Double-Haploid Maize Plants One Generation After the Chromosomes Doubling
}

\author{
R. M. O. Pires ${ }^{1}$, B. G. Ribeiro ${ }^{1}$, F. O. Bustamante ${ }^{2}$, E. V. R. Von Pinho ${ }^{1}$, H. O. Santos ${ }^{1} \&$ R. G. Von Pinho \\ ${ }^{1}$ Department of Agriculture, Universidade Federal de Lavras, Lavras, Brazil \\ ${ }^{2}$ Department of Genetic, Universidade Federal de Pernanbuco, Recife, Brazil \\ Correspondence: Raquel Maria de Oliveira Pires, Universidade Federal de Lavras, MG, Brasil. Tel: \\ 55-0359-9231-6031. E-mail: raquel.mopires@gmail.com
}

\author{
Received: February 18, 2019 Accepted: May 4, $2019 \quad$ Online Published: July 31, 2019 \\ doi:10.5539/jas.v11n11p41 URL: https://doi.org/10.5539/jas.v11n11p41
}

\begin{abstract}
The objectives in the present work were to identify maize double haploids one generation after chromosome duplication through the evaluation of phenotypic characteristics in thr field, flow cytometry and molecular markers SSR. The seeds used in the present study were obtained from a cross between four simple hybrids (DKB393, GNS 3225, GNS 3264, GNS 3032) and the KEMS inducer of gymnogenetic haploidy, used as a male parent. Seeds from this crossing were selected according to the R-Navajo marker and those considered haploid, were submitted to two different chromosome duplication protocols. Plants that survived to the chromosome duplication protocols were acclimatized in greenhouse and later transplanted to the field. After self-fertilization of the DH0 plants, the DH1 seeds obtained were seeded in the field, divided into treatments according to the parental and duplication protocols. At the vegetative stage V4 of the DH1 seedlings, leaf tissue samples were collected to identify ploidy via flow cytometry and DNA analyzes using microsatellite markers. These results were confronted with the morphological characteristics of the future DH1 plants developed in the field, evaluated with the use of descriptive tools. Statistical analyzes were performed using the generalized linear modeling approach and the exploratory and inferential analyzes of datas, by the use of graphical resources, barplot and boxplot. For the analysis of variance, were used the Student-Newman-Keuls test, and the Pearson's correlations. It was not observed uniformity of phenotypic characteristics of plants subjected to duplication protocols in the field and the use of descriptive tools in the morphological analysis of adult maize plants must be done carefully to avoid the wrong classification of determined genotypes related to the ployd. Flow cytometry must be used as screening in the identification of possible DH's and the molecular markers SSR can be used to prove the genetically inherited KEMS lineage and also to identify the double-haploid corn plants.
\end{abstract}

Keywords: double-haploid, induction, morphological analysis, molecular markers, SSR

\section{Introduction}

The search for greater productivity for crops today is the big challenge for plant breeders. In maize breeding programs, investments have been carried out with the objective of developing hybrids with characteristics that meet the farmers demands and the grain industry. However, the major biological limitation still consists of time for obtaining plants, since the exploration of the phenomenon of heterosis or hybrid vigor, is a costly process involving successive generations of self-fecundations (Ren, 2017).

In this sense, the double haploid-technology (DH) has gained importance for the development of hybrids in breeding programs, since it decreases the time for obtaining homozygote lineages in up to three generations, reduces costs and accelerates the introgression of traits of agronomic interest in genotypes of interest (Prigge et al., 2012).

The development of homozygote lineages allows better exploitation of genetic variability and increases the efficiency of selection. Plants in homozygosity will have the maximum additive variance, the effects of dominance neutralized, and the advantages in the selection of higher quantitative characteristics (Melchinger et al., 2013). In addition, the decrease of the costs with labor, use of smaller experimental area and anticipation of profits in commercial programs for maize breeding have made this technique a great success. 
However, the applicability of this methodology is still dependent on a few factors such as the use of inductors with high capacity of induction, efficient and reproducible protocols of chromosome doubling, as well as a precise system of identification and differentiation of diploid and haploid seeds (Chaikam et al., 2017).

Haploid seeds, generated through the use of stimulative lineages, are separated from diploid seeds using a combination of dominant markers present in the endosperm, embryo and radicle (Nanda \& Chase, 1966; Chase, 1963; Röber, 1999; Eder \& Chalyk, 2002). In the methodology of double haploids, it is common to use the marker gene R1navajo in identification of haploid seeds (Chase \& Nanda, 1965, 1966). The dominant allele R1-nj, of the regulator gene of anthocyanin in seed R1, allows the seeds to be selected visually. However, this selection is not always reliable once the marker may present incomplete penetrance and variable expressivity (Belicuas et al., 2007).

There are few works related to chromosome doubling in maize (Couto et al., 2013, 2015) and often the reported protocols upon being used, have low efficiency. In addition, studies that confirm the DH condition of duplicate materials in the field are nonexistent.

Thus, the objective of this work was to identify maize double haploid of a generation after the chromosome doubling through the evaluation of phenotypical characteristics in the field, flow cytometry and SSR molecular markers.

\section{Material and Methods}

The seeds used in this study were obtained from an experiment developed earlier by Couto et al. (2013) through the cross among four simple hybrids (DKB393, GNS 3225, GNS 3264, GNS 3032) and the gynogenetic haploid inducer KEMS (used as male parental), in which the seeds with purple endosperm and white embryo, originated from such crosses, were considered haploid in accordance with Navajo R marker (Chase \& Nanda, 1965).

The authors subjected the haploid seeds to two protocols of chromosome doubling, being that the plants that survived in the field, called DH0, which produced pollen and had style-stigma in sync, were self-fecundated, resulting in the generation DH1.

Thus, in the present work, with the aim of assessing the maintenance of DH in future generations, the cobs DH1 were harvested and seeds were threshed and dried at ambient temperature until $12 \%$ of moisture content to have reached the point of physiological maturity. The seeds were then mixed and divided into treatments, as shown in Table 1. These seeds were then stored in cold chamber at $10^{\circ} \mathrm{C}$, until the completion of the following experiments.

Table 1. Treatments evaluated in the season 2014/2015, established in accordance with the parents and the protocols of chromosome doubling performed by Couto et al. (2013)

\begin{tabular}{lll}
\hline Identification of materials & & \\
\hline Treatment & Hybrids & Protocols \\
\hline 1 & DKB393 $\times$ KEMS & 1 \\
2 & DKB393 $\times$ KEMS & 2 \\
3 & GNS3225 $\times$ KEMS & 1 \\
4 & GNS3225 $\times$ KEMS & 2 \\
5 & GNS3264 $\times$ KEMS & 1 \\
6 & GNS3264 $\times$ KEMS & 2 \\
7 & GNS3032 $\times$ KEMS & 1 \\
8 & GNS3032 $\times$ KEMS & 2 \\
\hline
\end{tabular}

In the season 2014/2015, the DH1 seeds of each treatment were sown in the field, and the experimental design used was a randomized complete blocks with eight treatments and four replicates each.

The plots were composed by lines of 10 meters of length, with spacing of $80 \mathrm{~cm}$ between rows and between plants of $25 \mathrm{~cm}$, and sowing of a seed per hole. The fertilization at sowing was $450 \mathrm{~kg}$ of formulated 8-28-16 and the top-dressing fertilization was performed as recommended for the maize cultivation in the region. Weed control was carried out manually and on a weekly basis.

During the flowering, to prevent unwanted crosses, the cobs were protected with plastic bags, prior to the emission of the style-stigmata. All plants that produced pollen and which possessed style-stigma in sync were 
self-fecundated for maintenance and use of the seeds in future experiments. The seedlings DH1 were individually identified aiming at relating the results of flow cytometry and microsatellite SSR markers, with the morphological characteristics of the DH1 plants in the field.

Leaf tissue samples were collected in the vegetative stage V4 and the medial portion of the young leaves completely developed was cut into segments of approximately $3 \mathrm{~cm}$ in length and wrapped in aluminum foil. Due to the amount of material, the flow cytometry analyzes were carried out in various stages.

For the analyzes of flow cytometry, samples of young leaf tissue of maize were crushed under ice, in Petri plates containing $1 \mathrm{~mL}$ of ice-cold LB01 buffer for obtaining the nuclear suspension (Dolezel, 1997), to which were added $2.5 \mu \mathrm{L}$ of RNase and $25 \mu \mathrm{L}$ of propidium iodide $\left(1 \mathrm{mg} \mathrm{mL}^{-1}\right)$, being the species Vicia faba $(26.9 \mathrm{pg} / 2 \mathrm{C})$ used as an external standard of reference. The analyzes were performed using the flow cytometry FacsCalibur (Becton Dickinson), the histograms obtained by WinMDI software 2.8 (2009; available at http://www.cyto. purdue.edu/flowcyt/software/Winmdi.htm) and at least 10 thousand cores were analyzed. The determination of the ploidy level of each sample was performed by comparing the position of the G1 peak of the sample with the G1 peak of the external standard of reference.

Part of the leaf tissue of all genotypes evaluated by flow cytometry was also evaluated by the technique with the SSR marker. In addition, it was used as a control, leaf samples of two parents (the KEMS inducing lineage and the hybrid DKB393). The extraction was performed according to Doyle and Doyle (1997). After the precipitate is dried, it was diluted with $50 \mu \mathrm{L}$ of ultrapure water with $2 \mu \mathrm{L}$ of RNAse $\left(10 \mathrm{mg} \mathrm{mL}^{-1}\right)$, keeping the temperature of $37^{\circ} \mathrm{C}$ for 1 hour. The DNA was quantified in a spectrophotometer GE Nanovue.

For the gels revelation, the staining method with silver nitrate was used. After verification of the parents polymorphism, the polymorphic primer BNLG238 was used to detect the gymnogenetic haploids and the unduplicated diploids, detected in flow cytometry (Couto et al., 2013).

PCR reactions consisted of $20 \mathrm{ng}$ of DNA, $0.5 \mu \mathrm{M}$ of each primer, $100 \mu \mathrm{M}$ of each dNTP, $10 \mathrm{mM}$ Tris-HCl (pH 8.6), $50 \mathrm{mM} \mathrm{KCl}, 2 \mathrm{mM} \mathrm{MgCl}$ and $1 \mathrm{U}$ of the enzyme Taq polymerase, in a total volume of $25 \mu \mathrm{L}$. The amplifications used were carried out in accordance with the schedule: a step of initial denaturation at $94{ }^{\circ} \mathrm{C}$ for 3 minutes, followed by 10 cycles at $94{ }^{\circ} \mathrm{C}$ for 20 seconds, $65^{\circ} \mathrm{C}$ for 30 seconds and $72{ }^{\circ} \mathrm{C}$ for 20 seconds, with the reduction of temperature of annealing at $1{ }^{\circ} \mathrm{C}$ to each cycle until it reaches $56^{\circ} \mathrm{C}$, followed by over 20 cycles with the temperature of annealing at $56{ }^{\circ} \mathrm{C}$ and a final step at $72{ }^{\circ} \mathrm{C}$ for 5 minutes. The amplification products were separated in $10 \%$ acrylamide gel electrophoresis at $110 \mathrm{~V}$ for 90 minutes.

In addition, approximately after 120 days of the crop cycle, some morphological characters of all plants DH1 were evaluated, namely: plant height; height of insertion of the first ear; stem diameter; number of green leaves; number of ears per plant and number of seeds per plant. Qualitative characteristics such as color of leaves, abnormalities in the tassels and in the style-stigma and deformities of the plants, were also evaluated and added as remarks. The statistical analyzes were performed using generations as replications and the proportions observed were evaluated using the approach of generalized mixed linear models (MLGM), similar to the one proposed by Nunes, Morais and Bueno Filho (2004). In this case, the binomial MLGM was used with logit link function, as described below:

$$
\begin{gathered}
\frac{\mathrm{R}_{\mathrm{ijk}}}{\mathrm{p}_{\mathrm{ijk}}} \sim \frac{\operatorname{Binomial}\left(\mathrm{m}_{\mathrm{ij} \mathrm{k},}, \pi_{\mathrm{ijk}}\right)}{\mathrm{m}_{\mathrm{i}}} \\
\log \left(\frac{\pi_{\mathrm{ijk}}}{1-\pi_{\mathrm{ijk}}}\right)=\mu+\mathrm{p}_{\mathrm{i}}+\mathrm{h}_{\mathrm{j}}+\mathrm{g}_{\mathrm{k}}+\mathrm{ph}_{\mathrm{ij}}+\mathrm{p}_{\mathrm{ijk}}
\end{gathered}
$$

Where,

$\mathrm{R}_{\mathrm{ijk}} / \mathrm{p}_{\mathrm{ijk}}$ : proportion observed in the plot that received the protocol $\mathrm{i}$ on hybrid $\mathrm{j}$ and the generation $\mathrm{k}$ admitted conditionally, regardless the random effect of plot; $\mu$ : intercept; $p_{i}$ : fixed effect of protocol $i ; h_{j}$ : fixed effect of the hybrid $\mathrm{j}$; $\mathrm{g}_{\mathrm{k}}$ : fixed effect of generation $\mathrm{k}$; $\mathrm{ph}_{\mathrm{ij}}$ : fixed effect of the interaction of hybrid $\mathrm{j}$ with the protocol $\mathrm{i} ; \mathrm{p}_{\mathrm{ijk}}$ : random effect of the plot ijk, being $\mathrm{p}_{\mathrm{ijk}} \sim \mathrm{N}\left(0, \sigma_{\mathrm{p}}^{2}\right)$.

The MLGMs were adjusted with estimation of fixed and random effects, as well as the variance components, via the Restricted Maximum Likelihood function (REML). To do this, the Lme4 package was used of the program R (version 2.14) (Bates, Maechler, \& Bolker, 2011). The significance of the effects of the model was verified through analysis of "deviance", with the application of the statistical test of $\chi^{2}$ (chi-square), at $5 \%$ of probability (Cordeio and Demétrio, 2007). In the effects that showed significant differences, grouping was performed based on the Mahalanobis distance, by nearest neighbor method and established the cutoff point using the method of bootstrap resampling (Mourão Júnior, 2001). 
In the data exploratory and inferential analyzes, the barplot and boxplot were used as graphic resources through the $\mathrm{R}$ statistical program (version 2.7.1), with the objective of quantifying the ploidies in percentage per treatment and observe the variability of morphological characteristics within and among the treatments, in addition to determining the presence or absence of individuals with extreme characteristics (outliers).

The analysis of variance, the Student-Newman-Keuls test and Pearson correlations were performed using the statistical software GENES, at 5\% of significance (Cruz, 2013).

\section{Results and Discussion}

No significant differences were observed among the sources of variation assessed (hybrids, generations, protocols) for the amount of DH1 seeds harvested, nor even to the means adjusted by Mahalanobis grouping. The adjusted mean percentage of the quantity of DH1 seeds harvested from DH0 self-fecundated plants for four hybrids was $58 \%$ (data not shown).

The values in percentage of ploidies observed in each treatment by flow cytometry technique can be visualized in Figure 1. All the ploidies were characterized by means of comparison of plants with the peak of the external standard used (Figure 2).

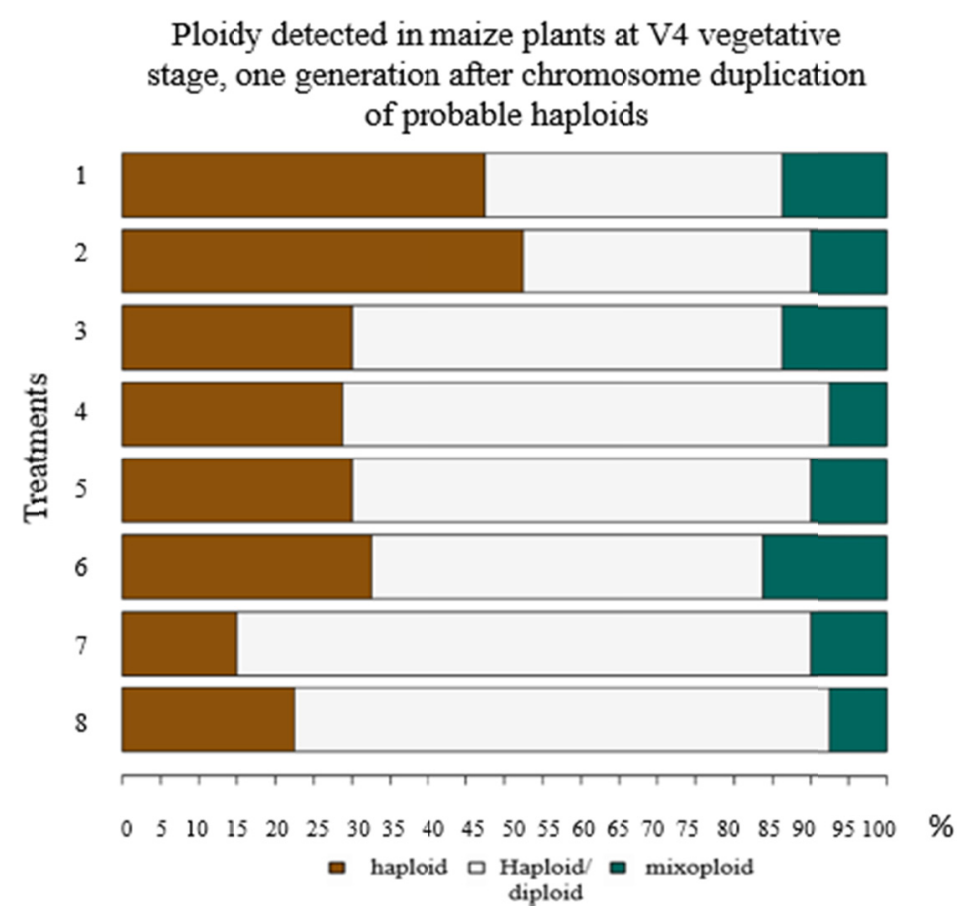

Figure 1. Barplot of values in percentage of ploidies detected in the vegetative stage V4 of the different hybrids in each treatment; 1-DKB393xKEMS/Prot 1, 2-DKB393xKEMS/Prot 2, 3-GNS3225xKEMS/Prot 1, 4-GNS3225xKEMS/Prot 2, 5-GNS3264xKEMS/Prot 1, 6-GNS3264xKEMS/Prot 2, 7-GNS3032xKEMS/Prot 1, 8-GNS3032xKEMS/Prot 2 depending on the total value of DH1 seeds sown in the field 

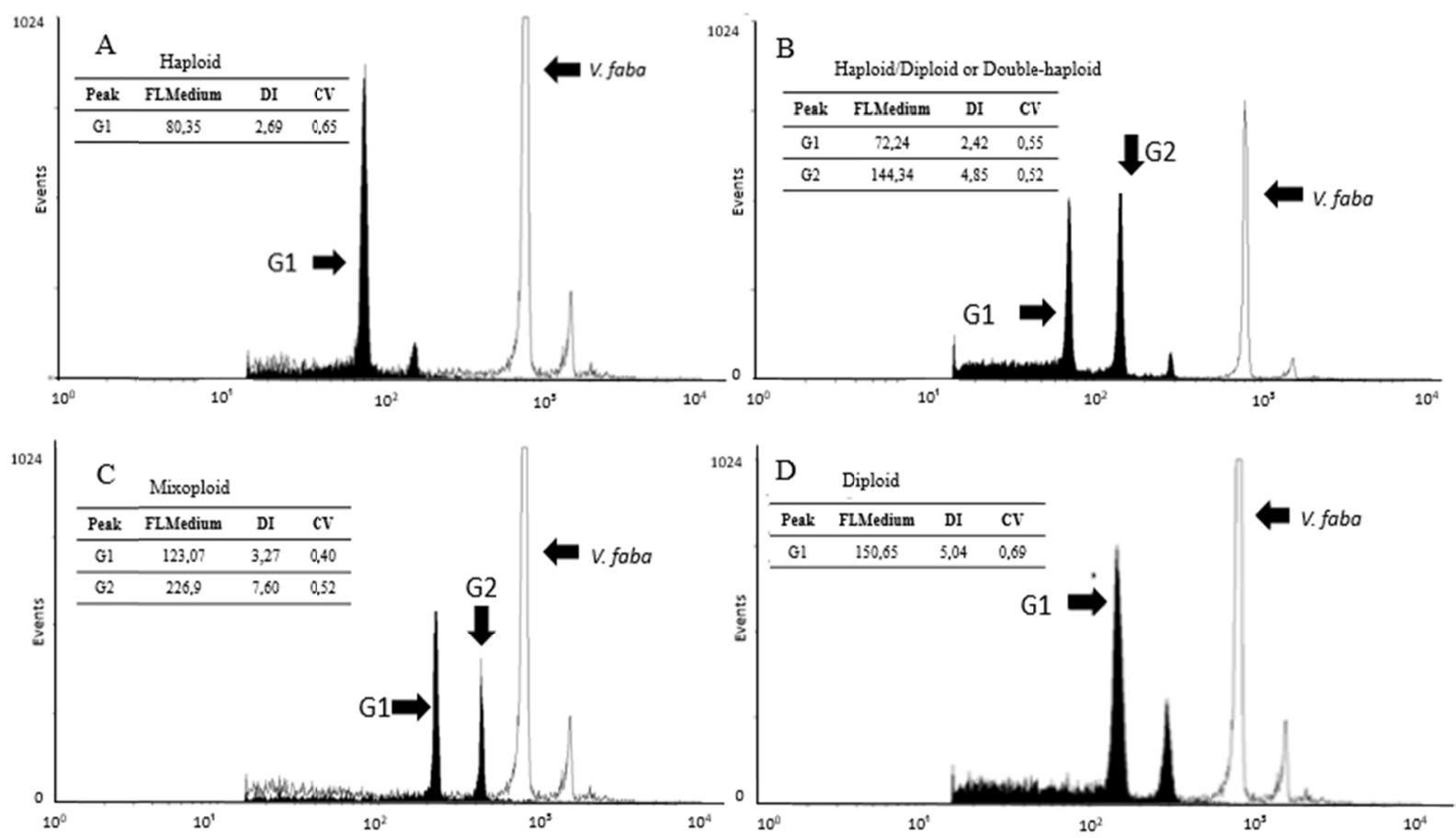

Figure 2. Histograms of DH1 seedlings of maize in the vegetative stage V4, a generation after chromosome doubling of probable haploids. A) haploid, B) haploid/diploid or double haploid, C) mixoploid and D) DKB genitor, diploid; vertical axis = number of cores read; horizontal axis = relative intensity of fluorescence. The arrows show the peaks (G1 and G2 and the external standard of reference $(V . f a b a)$ )

In all treatments, three distinct classes of ploidies were observed with emphasis to the high percentage of haploid plants in all of them. Considering that the environmental conditions were the same for all treatments installed in the field, thereby diminishing the environmental effect and considering the action of colchicine, two possibilities may be studied regarding these haploid values found even after a generation of inbreeding. The first concerns the process of artificial duplication, cited by many authors as low, inefficient and often irreproducible (Belicuas, 2007), in which factors such as time of exposure to the doubling agent and concentration, impede the restoration of the diploid condition and keep the seedlings in the haploid condition. This was observed in the present study, once the protocols used were still in the testing phase as regards their efficiencies.

Another possibility is that the process of chromosome doubling using colchicine was limited by the appearance of chimeras. Not always all the cells of the treated tissue polyploidize, which can lead to the formation of chimeras, i.e., tissues or plants with duplicate sectors and others not duplicate, which explains the high number of postoperative haploid duplication in the present study, as well as the high number of mixoploid plants (Schifino-Wittermann \& Agnol, 2004). Similar results regarding the appearance of haploids and mixoploids after chromosome doubling were reported by Battistelli et al. (2012), and Couto et al. (2015).

According to Dooghe et al. (2011), the success of a procedure of chromosome doubling is at the boundary between the toxicity and efficacy, since low concentrations or short periods of exposure can result in high rates of survival, without promoting the chromosome doubling, or high concentrations and long periods of exposure in addition to result in mortality, can also cause the reduplication of chromosomes, leading to the formation of cells with undesirable levels of ploidies.

In function of the individual tracking, it was observed that in all treatments, many of the plants identified as haploid in the initial stage of the plant (V4) by flow cytometry technique, died before completing the culture cycle. The losses were significant with values ranging from $26 \%$ in the treatment 4 to $76 \%$ in the treatment 1 . The values regarding to losses in relation to the preceding stage V4, are demonstrated in the graphic in negative values, indicating the reduction verified between the two assessments (Figure 3).

The plants that did not die presented morphological characteristics such as: late development in relation to the other plants, grain production in the tassel, phenomenon known as "tassel seeds" (Figures 4A and 4B) and lighter green leaves (Figure 4C). Belicuas et al. (2007) described that the haploids in the field showed fragile, brittle 
leaves and arrangement of leaves different when compared to a diploid individual, which was not observed in the present study.

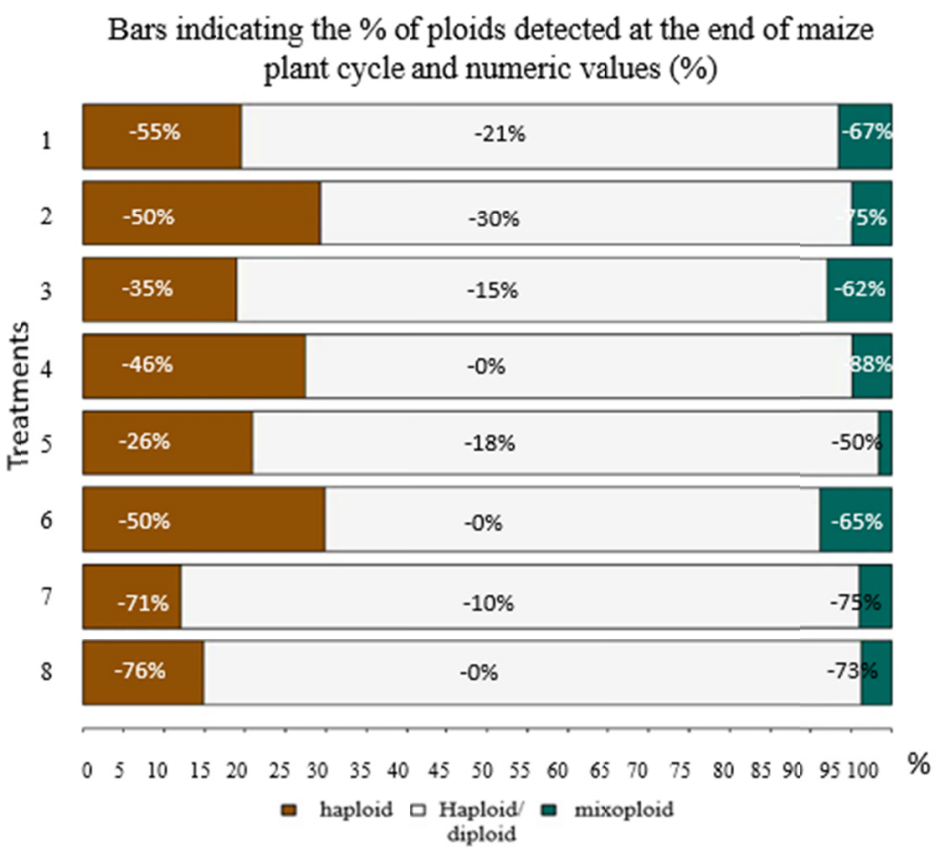

Figure 3. Barplot of values in percentage of ploidies detected in the final culture cycle of maize of different hybrids in each treatment; 1-DKB393xKEMS/Prot 1, 2-DKB393xKEMS/Prot 2, 3-GNS3225xKEMS/Prot 1, 4-GNS3225xKEMS/Prot 2, 5-GNS3264xKEMS/Prot 1, 6-GNS3264xKEMS/Prot 2, 7-GNS3032xKEMS/Prot 1,

8-GNS3032xKEMS/Prot 2, in function of the quantity of DH1 plants which survived in the field. Numeric values of the reduction (in percentage) of plants in relation to the vegetative stage V4

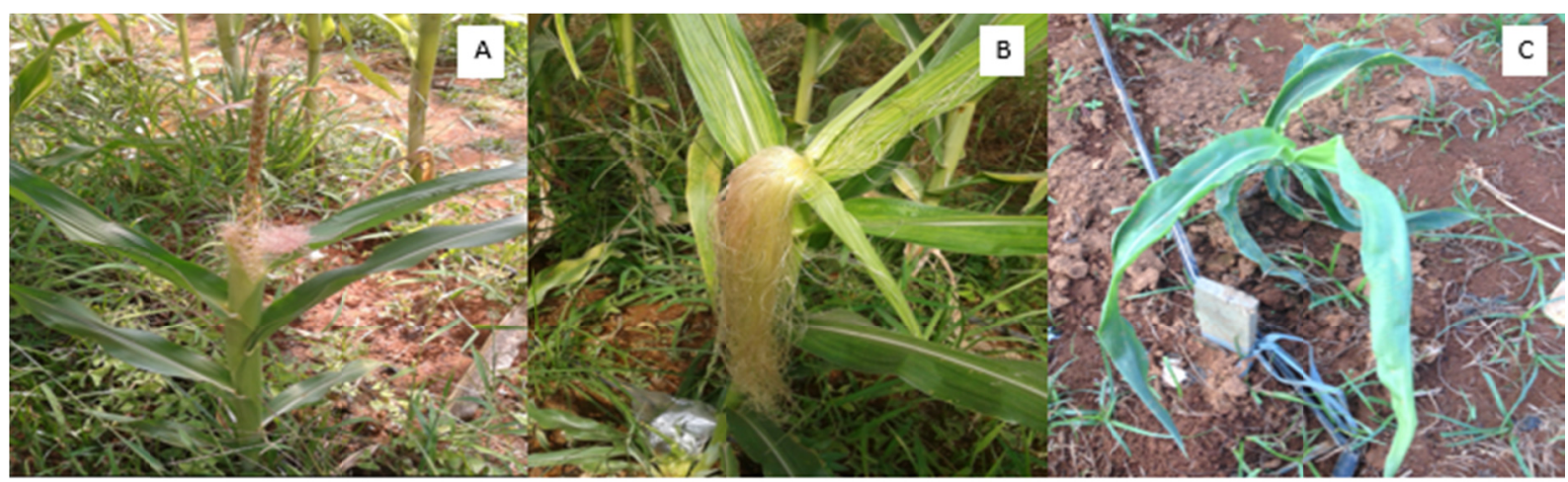

Figure 4. Photographs of the author demonstrating abnormalities of surviving haploid plants. A) and B) late development in relation to the other plants, grain production in the tassel, phenomenon known as "tassel seeds" and C) lighter green leaves

Soon after the chromosome doubling of hybrids used in the present study, performed by Couto et al. (2015), the authors observed that the different genotypes subjected to the same protocol of chromosome doubling showed similar behavior, in the same way that the behavior within each genotype, making it difficult for the identification of DH. Batistelli et al. (2013) suggest the use of molecular markers or observation of the segregation among the plants after planting to determine if a plant identified as $\mathrm{DH}$, is in fact a haploid genotype which was duplicated. In case there is segregation for the plant characteristics, it would be noticed that it was not about DH homozygous inbred. 
However, in the present study, the uniformity within the genotypes was observed only for some morphological characteristics such as: height of insertion of the first ear, number of green leaves and number of seeds (Figures $5 \mathrm{~B}, 5 \mathrm{D}$ and $5 \mathrm{~F}$ ), which showed a symmetric distribution of data in relation to the median, which characterizes less variability. On the other hand, plant height and stem diameter showed greater variability (Figures $5 \mathrm{~A}$ and 5C).

Many haploid plants had similar characteristics to the haploid/diploid plants considered mainly in relation to the characteristic plant height. In Figure 5A, through the boxplot in treatment 1, for example, there was only one plant discrepancy in relation to the average, for a total of eight haploids identified by flow cytometry. A similar situation was observed in treatments $2,3,5,6$ and 7 , in which few discrepant values were verified, in comparison with the actual values of haploid identified by flow cytometry.

The same was observed in the plants previously identified as polyploids/mixoploids. Plants with gene dosage higher than expected for a material that has been duplicated, usually have the "giga" effect, which is the increase in the size of the cells, due to the larger nuclear volume, and consequently have a higher height (Sattler, 2016). This fact can be observed in Figure 5A, where only the treatment six presented discrepant characters above the average, which disagrees with what was identified by flow cytometry through which more than 3 mixoploids/polyploids were accounted in each treatment. These observations contradict Batistelli et al. (2013), who suggest that the plants vigor would be a potential characteristic in the differentiation of ploidies in more advanced stage of development when the induced genotypes are simple hybrids.
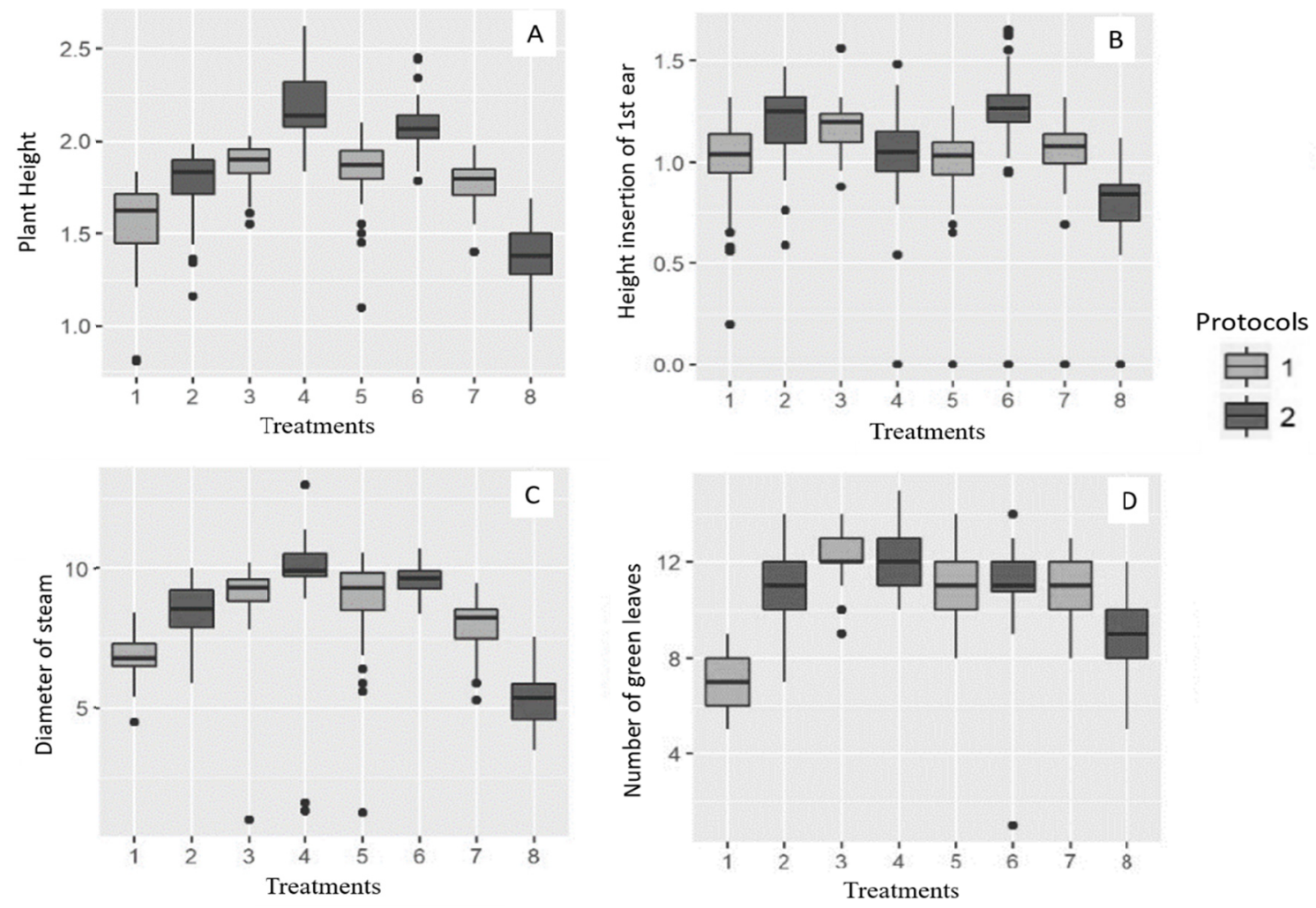

Figure 5. Boxplots for exploratory and inferential assessment of six morphological characteristics of maize plants (A-plant height, B-height of insertion of the first ear, C-stem diameter, D-numbers of green leaves per plant and number of ears per plant and F-number of seeds per plant), from four genotypes subjected to two protocols of duplication (1-DKB393xKEMS/Prot 1, 2-DKB393xKEMS/Prot 2, 3-GNS3225xKEMS/Prot 1, 4-GNS3225xKEMS/Prot 2, 5-GNS3264xKEMS/Prot 1, 6-GNS3264xKEMS/Prot 2, 7-GNS3032xKEMS/Prot 1,

8- GNS3032xKEMS/Prot 2). The box represents $50 \%$ of the data; the center mark in the box indicates the median and the points outside of the dimensions comprised by the box, the outliers. The test for the evaluation of overlap of outliers was not used in this experiment

Similar behaviors have been identified for the other characteristics in which there is a high correlation with the plant height (Table 2). For the quantity of seed harvested, for example (Figure 5F), it was observed that the 
treatment 4 , which thirteen haploid plants were counted and one plant per polyploid through flow cytometry, descriptively by the boxplot presented similar characteristics to the haploid/diploid plants, since haploids, known by the high degree of sterility, contradictorily produced a high number of seeds (13 haploid plants, 10 presented fertile tassel and were self-fecundated. In the literature, authors highlight that haploid plants can produce fertile tassel. Thus, the evaluated haploid plants were male-fertile plants (Chase, 1974; Geiger \& Schönleben, 2011), producing also fertile pollen.

Table 2. Pearson correlation coefficients among the morphological characteristics observed in maize hybrids subjected to two protocols of chromosome doubling, determined based on the average of the treatments

\begin{tabular}{lllllll}
\hline Phenotypic Correlations & \multicolumn{5}{l}{} \\
\hline & High & Insertion & Diameter & Leaves & Ears & Seeds \\
\hline High & 1 & 0.6542 & $0.9617^{* *}$ & $0.7621^{* *}$ & $0.814^{* *}$ & $0.8694^{* *}$ \\
Insertion & & $1^{* *}$ & $0.7529^{* *}$ & $0.5356^{* *}$ & $0.2748^{* *}$ & $0.2082^{* *}$ \\
Diameter & & $1^{* *}$ & $0.7786^{* *}$ & $0.8086^{* *}$ & $0.6129^{* *}$ \\
Leaves & & & $1^{* *}$ & $0.6287^{* *}$ & $0.6313^{* *}$ \\
Ears & & & & $1^{* *}$ & $0.5905^{* *}$ \\
Seeds & & & & & $1^{* *}$ \\
\hline
\end{tabular}

Note. ${ }^{*}$ Significant at $5 \%$ probability by the test.

Other considerations can be done by observing the data distribution in graphs boxplots. One of them relates to differences among the genotypes for each morphological evaluated characteristic. This distinction, confirmed by the analysis of variance in Table 3 is more expressive for the characteristic plant height (Figure 5D), where it was observed by the Student-Newman-Keuls test at the level of 5\%, significant differences among all the studied genotypes, being the genotype GNS3225xKEMS subjected to protocol 2 considered of greater average height and the genotype GNS3032xKEMS also subjected to Protocol 2, as the lowest average height. This same trend was observed for the variable diameter of stem, being that for these two characteristics there was the highest correlation (Table 2).

Table 3. Height of plant $(\mathrm{cm})$; height of insertion of the first ear $(\mathrm{cm})$; stem diameter $(\mathrm{cm})$; number of green leaves per plant, number of ears per plant and number of seeds per plant, of maize plants DH1 evaluated in the field from the four genotypes subjected to two protocols of duplication (1-DKB393xKEMS/Prot 1, 2-DKB393xKEMS/Prot 2, 3-GNS3225xKEMS/Prot 1, 4-GNS3225xKEMS/Prot 2, 5-GNS3264xKEMS/Prot 1, 6-GNS3264xKEMS/Prot 2, 7-GNS3032xKEMS/Prot 1, 8-GNS3032xKEMS/Prot 2)

\begin{tabular}{lllllll}
\hline \multirow{2}{*}{ Genotypes } & \multicolumn{5}{c}{ Morphological Characteristics } \\
\cline { 2 - 6 } & High & Insertion & Diameter & Leaves & Ears & Seeds \\
\hline $1^{* *}$ & $1.57 \mathrm{~g}$ & $1.02^{* *}$ & $6.86^{* *}$ & $7.01^{* *}$ & $1.06^{* *}$ & $23.22^{* *}$ \\
$2^{* *}$ & $1.74^{* *}$ & $1.21 \mathrm{a}$ & $8.64^{* *}$ & $11.01^{* *}$ & $1.25^{* *}$ & $23.15^{* *}$ \\
$3^{* *}$ & $1.81^{* *}$ & $1.17^{* *}$ & $9.12^{* *}$ & $12.21 \mathrm{a}$ & $1.32^{* *}$ & $91.17^{* *}$ \\
$4 * *$ & $2.19 \mathrm{a}$ & $1.05^{* *}$ & $9.79 \mathrm{a}$ & $12.01 \mathrm{a}$ & $1.69 \mathrm{a}$ & $150.74 \mathrm{a}$ \\
$5^{* *}$ & $1.85^{* *}$ & $1.01^{* *}$ & $8.94^{* *}$ & $10.80^{* *}$ & $1.65 \mathrm{a}$ & $41.79^{* *}$ \\
$6^{* *}$ & $2.07^{* *}$ & $1.25 \mathrm{a}$ & $9.56 \mathrm{a}$ & $11.22^{* *}$ & $1.41^{* *}$ & $104.27^{* *}$ \\
$7^{* *}$ & $1.68^{* *}$ & $1.06^{* *}$ & $7.94^{* *}$ & $11.17^{* *}$ & $1.14^{* *}$ & $63.64^{* *}$ \\
$8^{* *}$ & $1.38^{* *}$ & $0.79^{* *}$ & $5.31^{* *}$ & $8.90^{* *}$ & $1.05^{* *}$ & $50.72^{* *}$ \\
$\mathrm{CV}(\%)$ & $8.10^{* *}$ & $15.91^{* *}$ & $13.16^{* *}$ & $12.92^{* *}$ & $33.00^{* *}$ & $41.45^{* *}$ \\
\hline
\end{tabular}

Note. Column means followed by the same letter do not differ from each other by the Student-Newman-Keuls test at $5 \%$ probability.

Another important feature upon observing the morphological variables in Figure 5, are the graphics boxplots of those genotypes subjected to the same protocols of duplication that showed no linearity, i.e., there were not similar among themselves. This statement again contradicts Couto et al. (2015), who suggest similar behavior of those genotypes subjected to the same protocol of chromosome doubling. 
By what was shown above the verification of uniformity of individuals by means of morphologic markers of plants in the adult stage, must be done carefully, since it can lead to misclassification of certain genotypes in relation to ploidy level. In addition, the confirmation based on morphological characters of plants previously identified as double haploids (haploid/diploids) by means of flow cytometry, through observations of uniformity and segregation of individuals, is hampered by the high number of plants in the field, as well as by the similarity of the DH characteristics, with diploid plants. Thus, the confirmation of the origin of the plant, if hybrid or $\mathrm{DH}$, would be better detected by means of segregation of these materials.

With the aim of obtaining a more efficient confirmation of maintenance of DH a generation after the event of chromosome doubling, analyzes were carried out with microsatellite markers. Therefore, it was observed that the polymorphic primer BNLG238 was efficient to confirm the gymnogenetic inheritance of the lineage KEMS, since the haploid due to being homozygote subjects have only a band in the gel, originated from the hybrid parent. Furthermore, it was possible to confirm the presence of the double haploids, since they also have a gymnogenetic inheritance, thus presenting, just a band (Figure 6).

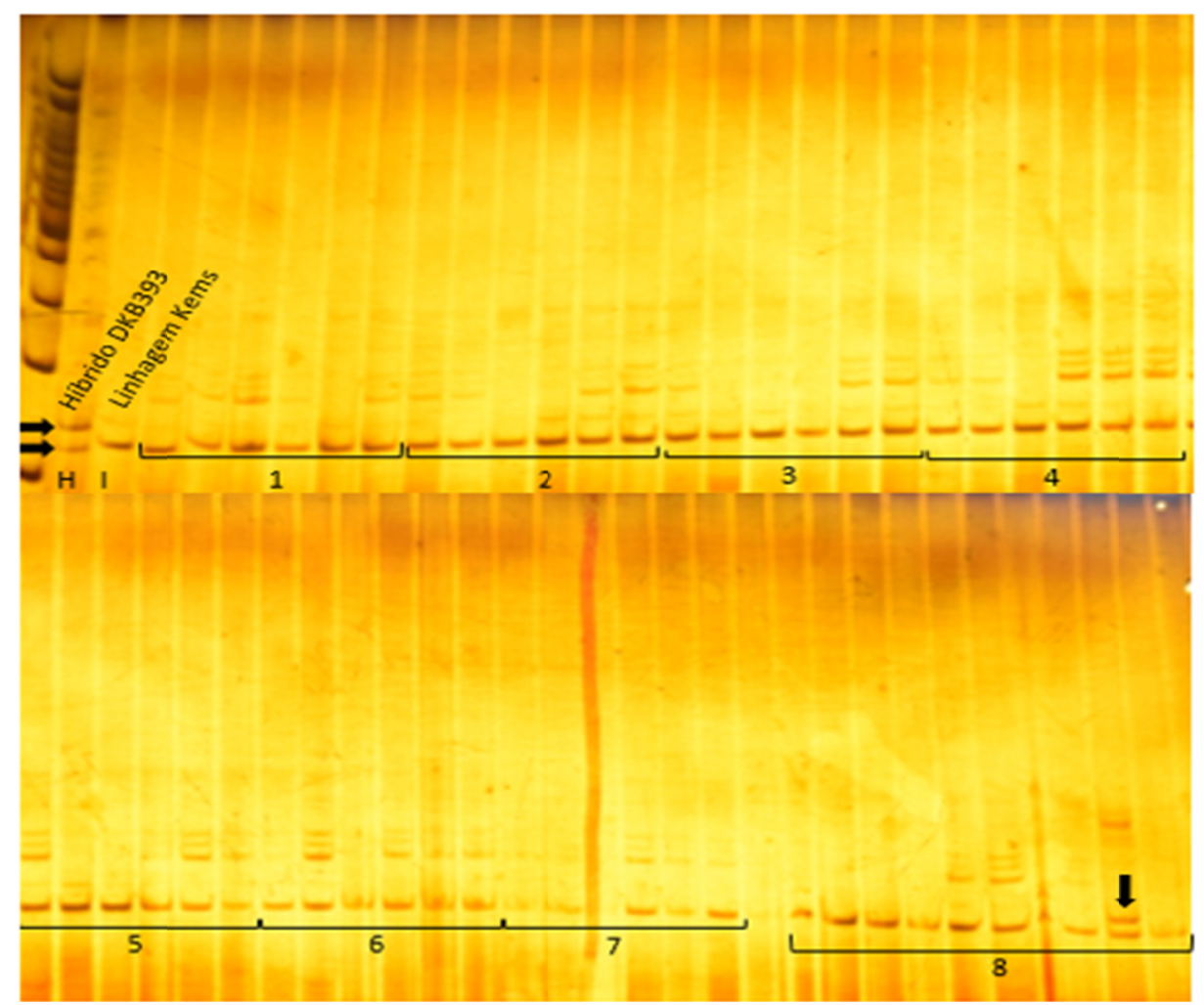

Figure 6. Electrophoretic pattern of products of amplification of the polymorphic primer BNLG 1238. (h) parent DKB393, I) inducing lineage of haploidy KEMS, 1, 2, 3, 4, 6, 6, 7, 8) Double- haploid confirmed in treatments 1, $2,3,4,5,6,7$ and 8 respectively. The arrow indicates the presence of a diploid represented by two bands

Couto et al. (2012) also used microsatellite markers to prove the androgenetic inheritance of haploids originated from the cross of a hybrid tropical with the lineage W23 in maize. The microsatellite markers have been used successfully in research with plants subjected to chromosome doubling of different species (Battistelli, 2012; BASTOS, 2016; Pereira et al., 2016). Thus, the microsatellite markers, because are codominant, allow for easy visualization and identification of homozygous individuals. Thus, it is possible to distinguish the diploids, which are heterozygous, from the haploids and double-haploids, that are homozygous.

Information concerning the quantity of double haploid seeds obtained are scarce and the companies and institutions that dominate this technology, choose not to disclose their results. In this sense, with the aim of providing more information, the number of ears harvested by treatment confirmed as double haploid plants by flow cytometry was quantified (Table 4). The value found is significantly high, totaling 118 ears, being that the highest number was observed in the treatments subjected to protocol 2 of duplication. These results reinforce the need of a generation of inbreeding in obtaining a greater quantity of double haploid seeds in breeding programs. 
Table 4. Number of DH1 maize plants considered double-haploids by flow cytometry and number of ears harvested at the end of 120 days of culture in field. (1-DKB393xKEMS/Prot 1, 2-DKB393xKEMS /Prot 2, 3-GNS3225xKEMS /Prot 1, 4-GNS3225xKEMS /Prot 2, 5-GNS3264xKEMS /Prot 1, 6-GNS3264xKEMS /Prot 2, 7-GNS3032xKEMS /Prot 1, 8-GNS3032xKEMS /Prot 2)

\begin{tabular}{lll}
\hline Treatment & $\begin{array}{l}\text { Number of plants considered double } \\
\text { haploids by flow cytometry }\end{array}$ & The number of DH ears harvested \\
\hline $1^{* *}$ & $44^{* *}$ & $8^{* *}$ \\
$2^{* *}$ & $42^{* *}$ & $22^{* *}$ \\
$3^{* *}$ & $35^{* *}$ & $9^{* *}$ \\
$4^{* *}$ & $48^{* *}$ & $19^{* *}$ \\
$5^{* *}$ & $42^{* *}$ & $11^{* *}$ \\
$6^{* *}$ & $46^{* *}$ & $16^{* *}$ \\
$7^{* *}$ & $27^{* *}$ & $10^{* *}$ \\
$8^{* *}$ & $34^{* *}$ & $23^{* *}$ \\
Total & $318^{* *}$ & $118^{* *}$ \\
\hline
\end{tabular}

\section{Conclusions}

Flow cytometry can be used to obtain more information as well as the SSR molecular markers can be used in proving the gymnogenetic inheritance of lineage KEMS lineage and also in the identification of double- haploids of maize plants.

\section{Acknowledgments}

To CNPq, CAPES, Fapemig and Universidade Federal de Lavras.

\section{References}

BASTOS. (2016). Queria ver essa referência pra checar se foi em milho.

Bates, D., Maechler, M., \& Bolker, B. (2013). Lme4: Linear mixed-effects models using S4 classes (Version $0.999375-42)$.

Battistelli, G. M. (2012). Estratégias para obtenção e identificação de duplohaploides em milho tropical (Dissertação, Mestrado em Genética e Melhoramento de Plantas, Universidade Federal de Lavras, Lavras).

Battistelli, G. M., Von Pinho, R. G., Justus. A., Couto, E. G. O., \& Balestre, M. (2013). Production and identification of doubled haploids in tropical maize. Genetics and Molecular Research, 2(4), 4230-4242. https://doi.org/10.4238/2013.October.7.9

Belicuas, P. R. (2007). Androgenetic haploids and SSR markers as tools for the development of tropical maize hybrids. Euphytica, 156, 95-102. https://doi.org/10.1007/s10681-007-9356-z

Chaikam, V. (2015). Analysis of effectiveness of R1-nj anthocyanin marker for in vivo haploid identification in maize and molecular markers for predicting the inhibition of R1-nj expression. Theoretische und Angewandte Genetik, 128(1), 159-171. https://doi.org/10.1007/s00122- 014-2419-3

Chaikam, V., Lopez, L. A., Martinez, L., Burgueño, J., \& Boddupalli, P. M. (2017). Identification of in vivo induced maternal haploids in maize using seedling traits. Euphytica, 213, 177. https://doi.org/10.1007/ s10681-017-1968-3

Chase, S. S., \& Nanda, D. K. (1965). Comparison of variability in inbred lines and monoploid-derived lines of maize (Zea mays L.). Crop Science, 5(4), 275-276. https://doi.org/10.2135/cropsci1965.0011183X0005 $00030026 \mathrm{x}$

Cordeiro, G. M., \& Demétrio, C. G. B. (2007). Modelos lineares generalizados (p. 165). Santa Maria: UFSM.

Couto, E. G. de O. (2013). Identification of haploid maize by flow cytometry, morphological and molecular markers. Ciência e Agrotecnologia, 37(1), 25-31.

Couto, E. G. O., Von Pinho, E. V. R., Von Pinho, R. G., Veiga, A. D., Carvalho, M. R., Bustamante, F. O., \& Nascimento, M. S. (2015). Verification and characterization of chromosome duplication in haploid maize. Genetics and Molecular Research, 14(2), 6999-7007. 
Cruz, C. D. (2013). Genes-A software package for analysis in experimental statistics and quantitative genetics. Acta Scientiarum, 35(3), 271-276.

Dang, N. C. (2012). Inducer line generated double haploid seeds for combined waxy and opaque 2 grain quality in subtropical maize (Zea mays L.). Euphytica, 183(1), 153-160.

Dhooghe, E., Van Laere, E., Eeckhaut, T., Leus, L., \& Van Huylenbroeck, J. (2011). Mitotic chromosome doubling of plant tissues in vitro. Plant Cell, Tissue and Organ Culture, 104, 359-373.

Doležel, J. (1997). Applications of fl ow cytometry for the study of plant genomes. Journal of Applied Genetics, 38(3), 285-302.

Krzywinski, M., \& Altman, N. (2014). Points of Significance: Visualizing samples with box plots. Nature Methods, 11(2), 119-120.

Melchinger, A. E. (2014). In vivo haploid induction in maize: Identification of haploid seeds by their oil content. Crop Science, 54(4), 1497-1504.

Melchinger, A. E. (2016b). Colchicine alternatives for chromosome doubling in maize haploids for doubled-haploid production. Crop Science, 56(2), 559-569.

Melchinger, A. E., Schipprack, W., Würschum, T., Chen, S., \& Technow, F. (2013). Rapid and accurate identification of in vivo induced haploid seeds based on oil content in maize. Scientific Reports, Nature, 3, $1-5$.

Mourão Júnior, M. (2001). Avaliação de um critério probabilístico em análise multivariada de agrupamento (Cluster analysis), por meio de simulação Monte Carlo (Dissertação Mestrado em Estatística e Experimentação Agropecuária, Universidade Federal de Lavras, Lavras).

Nunes, J. A. R., Morais, A. R., \& Bueno Filho, J. S. S. (2004). Modelagem da superdispersão em dados binomiais por um modelo linear generalizado misto. Revista de Matemática e Estatística, 22(1), 55-70.

Pereira, R. C., Santos, N. de S., \& Bustamante, F. de O. (2016). Stability in chromosome number and DNA content in synthetic tetraploids of Lolium multiflorum after two generations of selection. Ciencia Rural.

Prasanna, B. M., Chaikam, V., \& Mahuku, G. (2012). Doubled haploid technology in maize breeding: Theory and practice. Mexico: CIMMYT.

Prigge, V. (2011). Doubled haploids in tropical maize: I. Effects of inducers and source germplasm on in vivo haploid induction rates. Crop Science, 51(4), 1498-1506.

Prigge, V. Schipprack, W., Mahuku, G., Atlin, G. N., \& Melchinger, A. E. (2012). Development of in vivo haploid inducers for tropical maize breeding programs. Euphytica, 185(2), 481-490.

Ren, J., Wu, P., Trampe, B., Tian, X., Lubberstedt, T., \& Chen, S. (2017). Novel technologies in doubled haploid line development. Plant Biotechnology Journal, 15, 1361-1370.

Röber, F. K., Gordillo, G. A., \& Geiger, H. H. (2005). In vivo haploid induction in maize-performance of new inducers and significance of doubled haploid lines in hybrid breeding. Maydica, 50(3/4), 275-283.

Rotarenco, V. (2010). New inducers of maternal haploids in maize. Maize Genetics Cooperation Newsletter, $84(3), 1-7$.

Sattler, M. C., Carvalho, C. R., \& Clarindo, W. R. (2016). The polyploidy and its key role in plant breeding. Planta, 243(2), 281-296. https://doi.org/10.1007/s00425-015-2450-X

Schifino-Wittmann, M. T., \& Dall'agnol, M. (2001). Gametas não-reduzidos no melhoramento de plantas. Ciência Rural, 31, 169-175. https://doi.org/10.1590/S0103-84782001000100028

Shatskaya, O. A. (1994). Mass induction of maternal haploids in corn. Maize Genetics Cooperation Newsletter.

Sleper, J. A., \& Bernardo, R. (2016). Recombination and genetic variance among maize doubled haploids induced from F1 and F2 plants. Theoretical and Applied Genetics. https://oi.org/10.1007/s00122016-2781-4

Thakur, P. (2010). Cold stress effects on reproductive development in grain crops: An overview. Environmental and Experimental Botany, 67(3), 429-443. https://doi.org/10.1016/j.envexpbot.2009.09.004

Tseng, Y. (2012). New metods for haploid selection in maize (Graduate Theses and Dissertations, Iowa State University). 
Zinn, E. K., Tunc-Ozdemir, M., \& Harper, J. F. (2010). Temperature stress and plant sexual reproduction: Uncovering the weakest links. Journal of Experimental Botany, 61(7), 1959-1968. https://doi.org/10.1093/ jxb/erq053

\section{Copyrights}

Copyright for this article is retained by the author(s), with first publication rights granted to the journal.

This is an open-access article distributed under the terms and conditions of the Creative Commons Attribution license (http://creativecommons.org/licenses/by/4.0/). 